
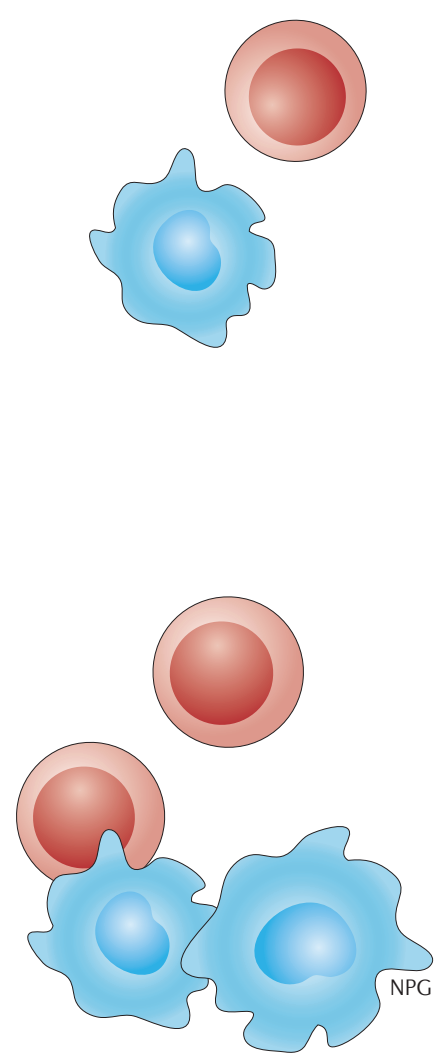

INFLAMMATION

\title{
Differential effects of salt on immune cell activity
}

\begin{abstract}
A high-salt diet has been linked to the development of hypertension and cardiovascular disease, and more recently to diseases caused by a disturbance in immune homeostasis, such as rheumatoid arthritis and multiple sclerosis (MS). Now, two studies published in The Journal of Clinical Investigation have shed light on the underlying mechanisms that link salt $(\mathrm{NaCl})$ intake to these diseases, by demonstrating differential effects of $\mathrm{NaCl}$ on the activation and function of various immune cells.

David Hafler and colleagues previously reported that high $\mathrm{NaCl}$ drives an increase in activation of pro-inflammatory type $17 \mathrm{~T}$ helper $\left(\mathrm{T}_{\mathrm{H}} 17\right)$ cells. In their latest study,
\end{abstract} the researchers investigated the effects of high $\mathrm{NaCl}$ on regulatory $\mathrm{T}\left(\mathrm{T}_{\mathrm{REG}}\right)$ cells, as defects in these cells are a major feature of autoimmune diseases. The effects of $\mathrm{NaCl}$ were examined both ex vivo and in a humanized mouse model. High $\mathrm{NaCl}$ was found to induce IFN $\gamma$ in $\mathrm{T}_{\mathrm{REG}}$ cells through serum/ glucocorticoid-regulated kinase

(SGK1). IFN $\gamma$

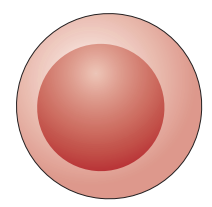
secretion was associated with loss of $\mathrm{T}_{\mathrm{REG}}$ cell function, which could be restored upon blockade or knockdown of IFN $\gamma$. The researchers propose that these new data provide a mechanistic link between an environmental factor and immune dysregulation in autoimmune disease, and that SGK1 might be a suitable therapeutic target.

"High-salt diets are associated with MS exacerbations, and patients with progressive MS have higher concentrations of $\mathrm{NaCl}$ in the central nervous system," explains Hafler. "Going forward, we have started a clinical trial to determine whether increases or decreases in $\mathrm{NaCl}$ intake influence immune system function in patients with MS."

Previous research from Katrina Binger, Dominik Muller and colleagues also showed that $\mathrm{T}_{\mathrm{H}} 17$ cells have boosted activation and function under high $\mathrm{NaCl}$ conditions. In their latest study, Binger et al. examined whether these findings could be translated to non-inflammatory immune cells. They focused on alternative (M2) macrophages, which are induced by IL- 4 and IL-13, and are involved in $\mathrm{T}_{\mathrm{H}} 2$ cell-mediated immunity and wound healing.

$\mathrm{C} 57 \mathrm{Bl} / 6$ mice were fed a high $\mathrm{NaCl}$ diet for 2 weeks and then injected intraperitoneally with chitin-an M2 macrophage-eliciting molecule. The researchers observed blunted expression of signature genes in macrophages isolated from the peritoneal cavity of these mice. Furthermore, mice on a high-salt diet had a delayed rate of wound healing. The researchers propose that non-inflammatory M2-macrophages have blunted activation and reduced function in response to $\mathrm{NaCl}$, which is in contrast to the stimulatory effects of $\mathrm{NaCl}$ on pro-inflammatory $\mathrm{T}_{\mathrm{H}} 17$ cells and $\mathrm{M} 1$ macrophages.

"When we put all of these studies together, the picture that is emerging is that salt is shifting the balance of the immune system," proposes Binger. "Salt is increasing the activity of pro-inflammatory cells, and the results from our current study and that of Hernandez et al. indicate that salt is also decreasing the activity of non-inflammatory immune cells."

Jessica K. Edwards

ORIGINAL ARTICLES Hernandez, A. L. et al.

Sodium chloride inhibits the suppressive function of FOXP $3^{+}$regulatory T cells. J. Clin. Invest. doi:10.1172/JCl81151|Binger, K. J. et al. High salt reduces the activation of IL-4- and IL-13stimulated macrophages. J. Clin. Invest. doi:10.1171//Cl80919 